\title{
Magnetic Flux Reversal in Laminated Ni-Fe Films
}

\author{
F. B. HUMPHREY, R. HASEGAWA, AND H. CLOW
}

\begin{abstract}
Anomalously fast flux reversal has been observed in films made of $\mathrm{Ni}-\mathrm{Fe}$ layers separated by $\mathrm{SiO}$. The speed of reversal increases as the number of identical layers of $\mathrm{Ni}-\mathrm{Fe}$ increases. For a 5-layer film, the anomalous speed is observed in films with the SiO thickness as great as $1600 \AA$. Reversal time curves presented as a family of curves of $1 / \tau=f\left(h_{\perp}\right)$ with $h_{s}$ as a parameter have two regions. The high-drive region has a lower slope in the laminated films when compared to the single-layer films. For this family of curves, a switching coefficient $S_{w o}$ can be defined, as the inverse slope, in a manner similar to the definition of $S_{w}$ for $1 / \tau=f\left(h_{s}\right)$ with $h_{\perp}$ as a parameter. For films with from two to five layers, $S_{w}{ }^{\prime}$ is constant at $1 \times 10^{-3} \mu \mathrm{S}$ and is smaller by an order of magnitude for the singlelayer films. A dual loop experiment is used to confirm that coherent rotation is not a dominant mechanism. It is concluded that a model must satisfy the following criteria to successfully describe flux reversal in the laminated films : It must provide rapid flux reversal for fields less than $H_{k}$, an insensitivity to transverse fields either constant or pulsed, and an interaction that can survive over a wide range of $\mathrm{SiO}$ thicknesses.
\end{abstract}

L AMINATED FILMS, i.e., layers of Ni-Fe separated by $\mathrm{SiO}$, exhibit what seems to be a characteristic and unique mode of flux reversal. It was previously shown that a film made of ten $200-\AA$-thick layers of $\mathrm{Ni}-\mathrm{Fe}(80-20)$, separated by nine $200-\AA$-thick layers of SiO, exhibited fast flux reversal, even for switching fields less than the anisotropy field $H_{k}$ [1]. Also, the curve of inverse reversal time as a function of longitudinal drive field did not exhibit a linear region, as do similar curves for other square loop materials. The family of $1 / \tau=f\left(H_{s}\right)$ curves was surprisingly insensitive to the magnitude of a static transverse field. It has also been demonstrated that this same laminated structure influences the wall structure, since the coercive force for laminated films is anomalously low [2]. It cannot be concluded, however, that the fast flux reversal is associated with an anomalously high wall mobility, since it has recently been shown that the wall mobility in laminated films is essentially the same as for a single-layer film and that the mobility is independent of the number of layers [3]. The investigation reported here concentrates on the higher drive fast flux reversal. An attempt is made to characterize the reversal and to establish the criteria for a flux reversal model that will describe reversal in laminated films.

The laminated films were fabricated in the same equipment and with the same procedure as the previous samples [1], [2]. The films were 1-cm diameter spots evaporated onto heated glass substrates $\left(320^{\circ} \mathrm{C}\right)$ in a pressure of about $10^{-5}$ Torr. The $80-20 \mathrm{Ni}-\mathrm{Fe}$ deposition rate was $25 \AA / \mathrm{s}$.

Manuscript received April 4, 1966. This paper was presented at the 1966 INTERMAG Conference, Stuttgart, Germany, April $20-22$.

F. B. Humphrey and R. Hasegawa are with the California Institute of Technology, Pasadena, Calif.

H. Clow is with the Research Laboratories, Electric and Musical Industries, Ltd., Hayes, Middlesex, England.
The $\mathrm{SiO}$ and $\mathrm{Ni}-\mathrm{Fe}$ layers were deposited alternately using a differential shutter. During the evaporation a control film was also deposited in such a way that it was screened from the $\mathrm{SiO}$ source. The resultant $\mathrm{Ni}-\mathrm{Fe}$ film was essentially a single-layer film of thickness identical to the total thickness of all magnetic layers. One series consisted of films with from one to five $350-\AA$-thick layers of $\mathrm{Ni}-\mathrm{Fe}$ separated by $100-\AA$-thick layers of $\mathrm{SiO}$, made identically. It should be noted that the control films for this series varied in thickness. Another series was made consisting of five $350 \AA$ thick layers of Ni-Fe separated by from 100 $\AA$ - to 1600 -A-thick layers of SiO. The thickness of the NiFe layers was measured by observing the spacing between the uniform mode and the first spinwave mode (at $X$ band) in a ferromagnetic resonance experiment. The $\mathrm{SiO}$ thickness was calculated by measuring the total thickness of the Tolansky interferrometric method and assuming all $\mathrm{SiO}$ layers were identical. Angular dispersion was measured by the method of Hasegawa et al. [8] to be $\alpha_{90}$ $<3^{\circ}$.

The flux reversal measurements were made in a $50-\Omega$ strip line section, with a center member $1.5-\mathrm{cm}$ wide to ensure a uniform field at the sample. The current pulse was obtained by discharging a cable through a coaxial relay with mercury wet contacts. The field pulse had a rise of less than 0.5 ns with a duration of $1 \mu \mathrm{s}$ and was continuously variable in amplitude. The flux change was observed with a single-turn loop around the center of the film arranged as a bridge in conjunction with another loop, with provision to balance the inductive voltage in both amplitude and time. The flux change was observed by integrating the induced voltage with an active integrator having a response time of $1.25 \mathrm{~ns}$, a decay time of $12 \mu \mathrm{s}$, and an effective gain of $1 \mathrm{mV}-\mu \mathrm{s}$ for a 0.5 -volt output. The output of the integrator was observed using a conventional sampling ascilloscope with a response time of $0.6 \mathrm{~ns}$. The reversal time $\tau$ was defined as the time from 10 to 90 percent of the integrated flux. Various coils were arranged around the sample to provide the reset field, transverse field, and earth's field cancellation. For each sample the anisotropy field was measured as $2 K / M$ on a torquemeter [4] and checked with a low-frequency ferromagnetic resonance spectrometer [5]. For the layered samples, the observed value of $H_{k}$ is an average of $H_{k}$ for each layer.

To demonstrate that the anomalous reversal times are indeed associated with the layered structure, nearly identical samples, differing only in the number of layers, were investigated. Reversal time as a function of drive was then observed for each, with the initial angle essentially zero; the results are shown in Fig. 1. The anomalous behavior becomes more marked as the number of layers increases. Since the slope cannot be used to compare speed, an indication of speed may be defined as $1 / \tau\left(\mu \mathrm{s}^{-1}\right)$ for $h_{s}=$ $H_{s} / H_{k}=1$. This parameter increases linearly from 2 to 140 


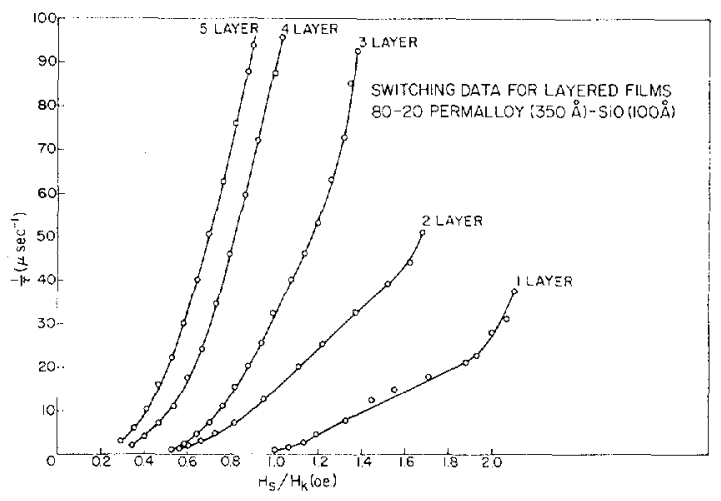

Fig. 1. Inverse of reversal time as function of normalized drive field with number of layers as parameter.

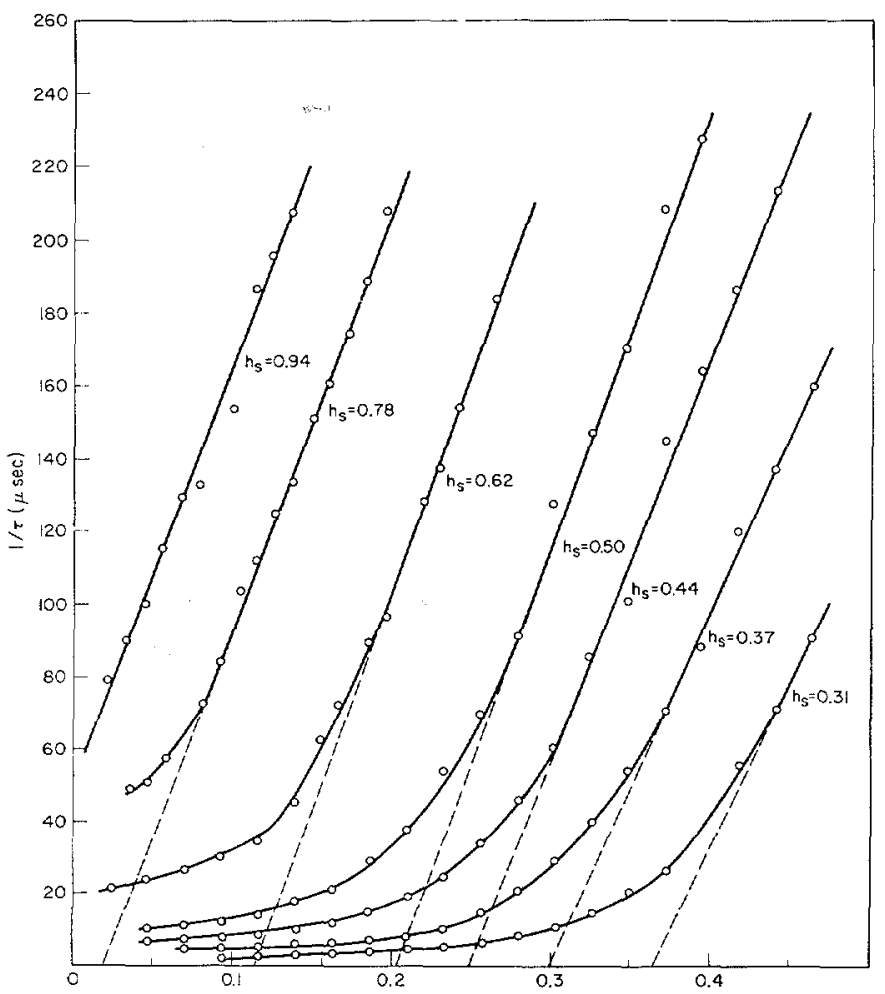

Fig. 3. Inverse of reversal time as function of normalized transverse field with normalized drive field as parameter for 3-layer laminated film.

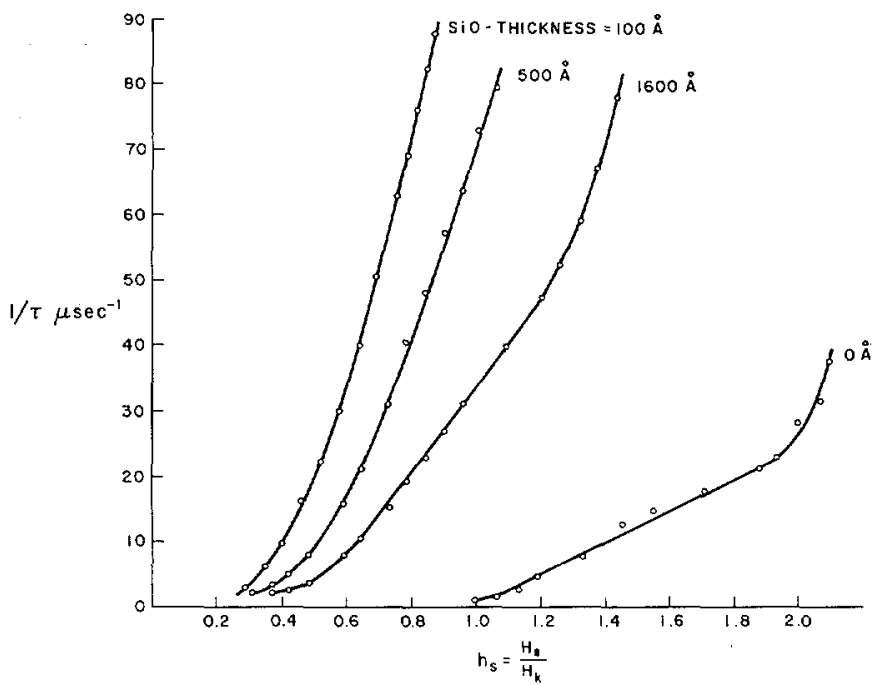

Fig. 2. Inverse of reversal time as function of normalized drive field with thickness of $\mathrm{SiO}$ layers for 5-layer laminated film.

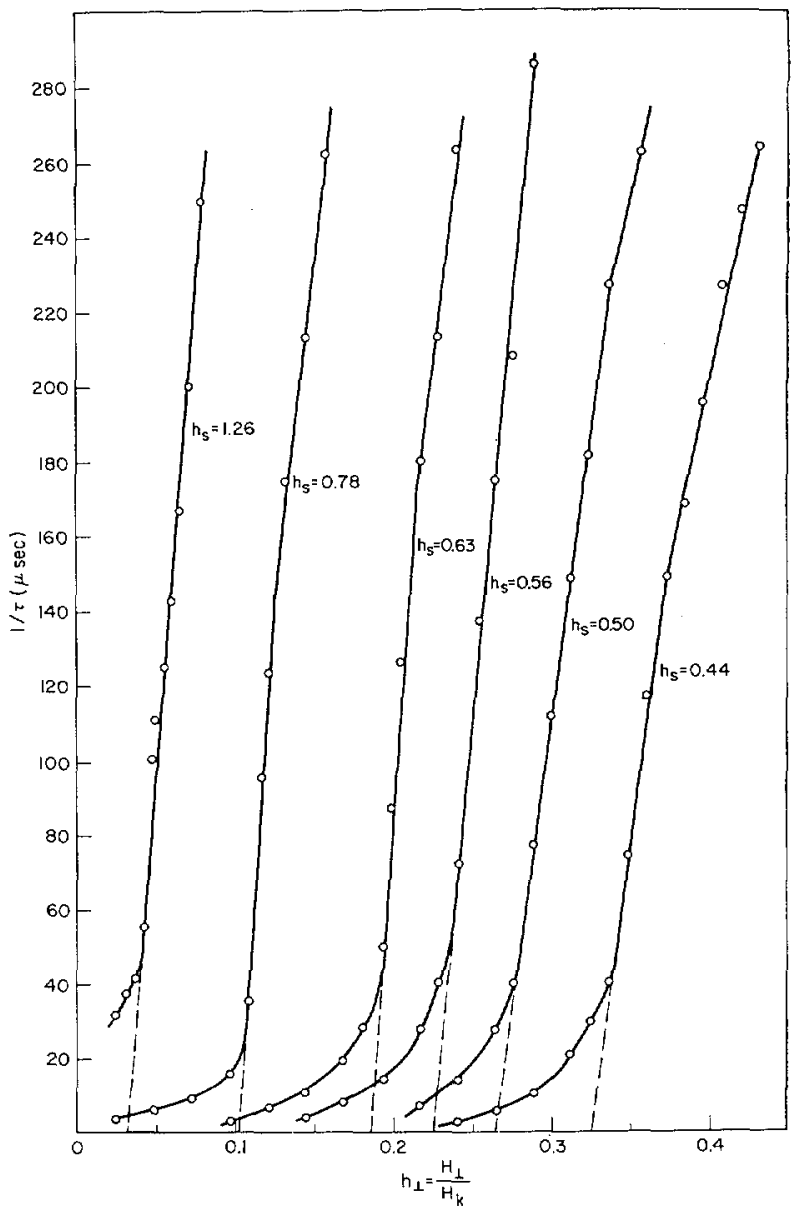

Fig. 4. Inverse of reversal time as function of normalized transverse field with normalized drive field as parameter for single-layer laminated film. Typical of all control films (this was the control film for the 3-layer laminated film). 


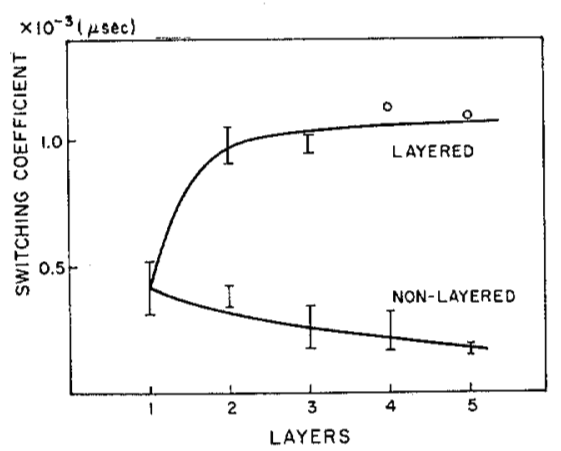

Fig. 5. Inverse slope from $1 / \tau=f\left(h_{\perp}\right)$ plot as function of number of layers. Control films shown will be of various thicknesses.

as the number of identical layers goes from 1 to 5 , respectively. Sensitivity to the thickness of the SiO layer is demonstrated by similar results shown in Fig. 2. This series of 5-layer films has the thickness of the SiO layer as a parameter; the anomalous behavior becomes less noticeable as the thickness of the SiO layer increases. On the basis of these three points, there seems to be a linear dependence of $1 / \tau\left(h_{s}=1\right)$ on the log of the SiO thickness with an extrapolation to a single-layer behavior at about 4000- $\AA \mathrm{SiO}$ thickness.

It has been suggested by Telesnin and Kolotov [6] that a more informative presentation of flux reversal data can be made by observing $1 / \tau=f\left(h_{\perp}\right)$ with $h_{s}$ as a parameter (rather than $1 / \tau=f\left(h_{s}\right)$ with $h_{\perp}$ as a parameter $\left(h_{\perp}=\right.$ $\left.H_{\perp} / H_{k}\right)$. Plots of $1 / \tau$ as a function of $h_{\perp}$ with $h_{s}$ as a parameter for the 3-layer laminated film can be seen in Fig. 3; a similar plot for the control film can be seen in Fig. 4. The high drive region for both films is linear. As the longitudinal field parameter $h_{s}$ is changed, the curves form a parallel set with the inverse slope of this set defined as the switching parameter $S_{w}{ }^{\prime}$ [similar to the usual switching parameter, but primed to indicate that it is from $1 / \tau=$ $f\left(h_{\perp}\right)$ ]. This switching parameter is plotted in Fig. 5 as a function of the number of layers. It can be seen that, for films with from 2 to 5 layers, $S_{w}{ }^{\prime}$ is constant at $1 \times 10^{-3}$ $\mu \mathrm{s}$, whereas, for the control films, $S_{w}{ }^{\prime}$ is smaller by an order of magnitude.

The high-drive linear region in normal films, as seen in Fig. 4, has been identified by Telesnin and Kolotov [6] as being a region in which the flux reversal mechanism is essentially coherent rotation. The break between the lower drive region and the higher drive linear region has been associated with the onset of this mode of flux reversal. Simultaneous observation of the flux in the longitudinal and transverse direction has been used as a definitive method to characterize coherent rotation [7]. The 5layer film (with 100- $\AA \mathrm{SiO}$ ) was observed with a dual loop, so that the longitudinal and transverse flux could be observed and compared. There was no noticeable relationship between the transverse and the longitudinal flux in the region where a large increase in transverse flux might be expected. The fraction of flux in a coherent mode increased smoothly over the entire range of $h_{\perp}$ and $h_{s}$; even for large values of $h_{s}$ and $h_{\perp}$, more than 50 percent of the flux was seldom obtained. This result clearly demonstrates another difference between single-layer and multilayer films, in that the laminated films reverse by some mechanism other than uniform rotation.

Even though the anomalously fast flux reversal is not completely understood the mechanism must be consistent with the following criteria. The model must be one which will provide rapid reversal for drive fields less than the anisotropy field. The mechanism must be such that the flux reversal time is insensitive to transverse fields either constant or pulsed. Finally, the model must provide an interaction that can survive over a wide range of $\mathrm{SiO}$ thicknesses. These criteria should provide the foundation for a description of this anomalously fast flux reversal.

\section{REFERENCES}

[1] F. B. Humphrey and H. Clow, "Fast flux reversal in laminated nickel-iron thin films," Nature, vol. 204, p. 769, 1964.

[2] H. Clow, "Very low coercive force in nickel-iron films," Nature vol. 194, p. $1035,1962$.

[3] C. E. Patton and F. B. Humphrey, "Domain wall motion in multi-layered magnetic thin films," 'J. Appl. Phys., Magnetics Conference Supplement, in Press.

[4] F. B. Humphrey and A. R. Johnston, "Sensitive automatic torque balance for thin magnetic films," Rev. Sci. Instr., vol. 34, p. $348,1963$.

[5] R. H. Kingston and P. E. Tannenwald, "Ferromagnetic resonance in UHF in thin films," $J$. Appl. Phys., vol. 29, p. 232, 1958.

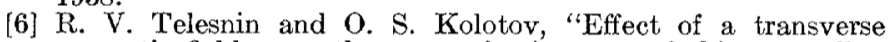
magnetic field upon the magnetization rate of thin permalloy films," Fiz. metal. metalloved., vol. 17, p. 834, 1964.

[7] F. B. Humphrey, "Transverse flux change in soft ferromagnetics," J. Appl. Phys., vol. 29, p. 284, 1958.

[8] R. Hasegawa, S. Uchiyama, and Y. Sakaki, "Measurement of anisotropy dispersion in thin ferromagnetic films," Japan $J$. A ppl. Phys., vol. 3, p. 671, 1964. 